\title{
A HIGH-FREQUENCY SEARCH FOR PULSARS WITHIN THE CENTRAL PARSEC OF Sgr A*
}

\author{
J.-P. Macquart ${ }^{1,2,6}$, N. KaneKar ${ }^{3,4,6,7}$, D. A. Frail ${ }^{3}$, And S. M. Ransom ${ }^{5}$ \\ ${ }^{1}$ Department of Astronomy, California Institute of Technology, Pasadena, CA 91125, USA; J.Macquart@ curtin.edu.au \\ 2 ICRAR/Curtin University of Technology, Bentley, WA 6845, Australia \\ ${ }^{3}$ National Radio Astronomy Observatory, 1003 Lopezville Road, Socorro, NM 87801, USA \\ ${ }^{4}$ National Centre for Radio Astrophysics, Tata Institute of Fundamental Research, Ganeshkhind, Pune-411007, India \\ ${ }^{5}$ National Radio Astronomy Observatory, 520 Edgemont Road, Charlottesville, VA 29903, USA \\ Received 2009 December 8; accepted 2010 April 4; published 2010 May 5
}

\begin{abstract}
We report results from a deep high-frequency search for pulsars within the central parsec of Sgr A* using the Green Bank Telescope. The observing frequency of $15 \mathrm{GHz}$ was chosen to maximize the likelihood of detecting normal pulsars (i.e., with periods of $\sim 500 \mathrm{~ms}$ and spectral indices of $\sim-1.7$ ) close to Sgr $\mathrm{A}^{*}$ that might be used as probes of gravity in the strong-field regime; this is the highest frequency used for such pulsar searches of the Galactic center (GC) to date. No convincing candidate was detected in the survey, with a $10 \sigma$ detection threshold of $\sim 10 \mu \mathrm{Jy}$ achieved in two separate observing sessions. This survey represents a significant improvement over previous searches for pulsars at the GC and would have detected a significant fraction ( $\gtrsim 5 \%)$ of the pulsars around Sgr A*, if they had properties similar to those of the known population. Using our best current knowledge of the properties of the Galactic pulsar population and the scattering material toward Sgr A*, we estimate an upper limit of 90 normal pulsars in orbit within the central parsec of Sgr A*.
\end{abstract}

Key words: Galaxy: center - pulsars: general

Online-only material: color figures

\section{INTRODUCTION}

The detection of radio-emitting neutron stars within the central parsec of our Galaxy would provide us with an unprecedented opportunity to study the super-massive black hole Sgr A* and its environs. For example, a single orbiting pulsar would yield a direct probe of the magneto-ionized accretion environment around a black hole through measurements of temporal changes in the dispersion and rotation measures (Cordes \& Lazio 1997). Pulsars orbiting within the curved spacetime around Sgr A* (with orbital periods of $\lesssim 100$ years) could serve as probes of gravity in the strong-field regime, at field strengths far larger than those accessible with neutron star binaries. The long-term timing of such pulsars, supplemented by accurate astrometry, would allow precise determination of their threedimensional orbital motion around Sgr A*. Depending on the properties of the pulsars and their orbits, it should be possible to measure subtle general relativistic deviations from Keplerian orbits (e.g., time dilation, gravitational redshifts, frame dragging, Shapiro delays, etc; e.g., Cordes et al. 2004; Pfahl \& Loeb 2004), and it may even be possible to determine the spin of the black hole (e.g., Wex \& Kopeikin 1999; Kramer et al. 2004).

While theoretical estimates indicate that $100-1000$ radio pulsars with periods $\lesssim 100$ years should be orbiting Sgr A* (Pfahl \& Loeb 2004), the observational evidence for neutron stars at the Galactic center (GC) is mostly indirect. For example, recent studies have found a number of dense clusters of young, massive stars within $\sim 1$ pc of Sgr A* (Schödel et al. 2003; Ghez et al. 2005; Paumard et al. 2006), while Wang et al. (2006) report X-ray observations of a pulsar wind nebula near the massive stellar complex IRS 13, with properties consistent with it being powered by a young neutron star. Long-term monitoring by Chandra has revealed an excess of transient sources within

\footnotetext{
6 NRAO Jansky Fellow.

7 Ramanujan Fellow.
}

a parsec of Sgr A*, interpreted by Muno et al. (2005) as a population of X-ray binaries. The flaring radio and X-ray source detected by Bower et al. (2005), 0.1 pc from Sgr A*, is also likely to be an X-ray binary.

Despite the above evidence for massive stars around Sgr A*, there is a remarkable dearth of radio pulsar detections there, despite several deep searches (e.g., Johnston et al. 1995; Johnston et al. 2006; Deneva et al. 2009). The closest known radio pulsars are $11^{\prime}$ from $\operatorname{Sgr} \mathrm{A}^{*}$, and less than $1 \%$ of the known pulsar population lies within a degree of the GC, despite indications of a large population in its environs (Deneva et al. 2009). The reason for this deficit is well understood: hyper-strong scattering of radio waves by the turbulent, ionized gas within the central $100 \mathrm{pc}$ of Sgr A*, which results in temporal smearing of pulsed signals. This pulse broadening has a strong frequency dependence, $\propto v^{-4}$, making it near-impossible to detect pulsars at the typical observing frequencies of $\lesssim 1.4 \mathrm{GHz}$ (e.g., Lazio \& Cordes 1998).

To overcome the effects of temporal smearing, searches for pulsars at the GC have been carried out at progressively higher observing frequencies over the last few years (e.g., Johnston et al. 2006; Deneva et al. 2009), albeit as yet without a detection in the central $25 \mathrm{pc}$. In this work, we report results from a deep Green Bank Telescope (GBT) search for pulsars toward Sgr A* at $\sim 15 \mathrm{GHz}$, the highest observing frequency used till date. The choice of this frequency is motivated in Section 2, and the observations and results described in Section 3. Finally, Section 4 discusses the constraints placed by our observations on the GC pulsar population, and the prospects for pulsar detections in future surveys.

\section{A PULSAR SEARCH STRATEGY FOR THE GALACTIC CENTER}

Previous surveys of the GC pulsar population have concentrated on maximizing the likelihood of detection within a few 
degrees of Sgr A* (e.g., Johnston et al. 2006; Deneva et al. 2009). This has the effect of lowering the optimal observing frequency, because a larger volume can be searched in a given amount of integration time at lower frequencies, due to the larger telescope beam. However, the region containing pulsars capable of probing gravitational effects in the strong-field regime is quite small, $0^{\prime \prime} 4$, much smaller than the GBT beam at even frequencies $\gg 10 \mathrm{GHz}$. Since our long-term goal is to use pulsars as probes of strong-field gravity around Sgr A*, we optimized our search strategy to detect pulsars in the immediate vicinity of (i.e., in orbit around) Sgr A* itself. Cordes \& Lazio (1997) have previously described the observational challenges in detecting periodic emission at the GC; their main arguments are summarized below.

The optimal observing frequency depends on three competing effects, the temporal scattering, the pulsar emission spectrum, and the telescope sensitivity, all with very different frequency dependences. For example, the generally steep spectral energy dependence of the pulsar emission $\left(S_{v} \propto v^{\alpha}\right.$, with the distribution centered on $\alpha \sim-1.7)$ drives searches to low frequencies, but competes against the strong $v^{-4}$ spectral dependence of temporal smearing, due to which pulsars are severely attenuated at frequencies below which the scattering time exceeds the pulse period. We note, in passing, that the intervening ionized plasma also introduces a dispersive delay in the pulsar arrival times which can lead to a smearing of the pulse in individual filter-bank frequency channels used to detect the signal, thus degrading pulse detectability. While this effect has been formally included in the present analysis, it is insignificant for the fine spectral resolution offered by modern filter banks, for the estimated dispersion measure of $\sim 1500-3000 \mathrm{pc} \mathrm{cm}^{-3}$ for the GC region (Lazio \& Cordes 1998)

The primary uncertainty in the temporal smearing time lies in the distance of the scattering screen from the GC. The best estimate of this distance is from Lazio \& Cordes (1998), who combined all known tracers of ionized gas (the scattering diameters of masers and $\mathrm{OH} / \mathrm{IR}$ stars, free-free emission, and absorption, etc.) in a maximum likelihood analysis to obtain $D_{\text {scat }}=133_{-80}^{+200} \mathrm{pc}$. Making the usual simplifying assumption that the scattering material is confined to a thin screen results in a temporal smearing timescale for pulsars near Sgr A* of

$$
\tau_{\mathrm{ISM}}=0.116\left(\frac{D_{\text {scat }}}{100 \mathrm{pc}}\right)^{-1}\left(\frac{v}{10 \mathrm{GHz}}\right)^{-4} \mathrm{~s} .
$$

At a given frequency, a pulsar with period smaller than $\sim \tau_{\text {scat }}$ would effectively have its pulses smeared into one another. In other words, for a given observing frequency, it is not possible to detect a pulsar whose period is much shorter than the temporal broadening time at that frequency (see Figure 1). For instance, a pulsar of period $50 \mathrm{~ms}$ is dominated by temporal smearing at frequencies $\lesssim 11 \mathrm{GHz}$, while the detection of a pulsar of period $5 \mathrm{~ms}$ is strongly hampered at frequencies $\lesssim 26 \mathrm{GHz}$. Combining these effects, we estimate the temporal smearing time for a pulse of intrinsic width $\tau_{\text {intrinsic }}$, and with contributions from scattering $\left(\tau_{\text {ISM }}\right)$, interstellar dispersion $\left(\tau_{\mathrm{DM}}\right)$, and temporal binning $\left(\tau_{\text {res }}\right)$ to the overall observed pulse width to be

$$
\tau_{\mathrm{obs}}=\left[\tau_{\text {intrinsic }}^{2}+\tau_{\mathrm{ISM}}^{2}+\tau_{\mathrm{DM}}^{2}+\tau_{\mathrm{res}}^{2}\right]^{1 / 2}
$$

This equation affords a sufficiently good approximation to the effects of temporal broadening, shown in detail in Figure 1, when neither the intrinsic pulse profile nor the temporal broadening kernel are known in detail.

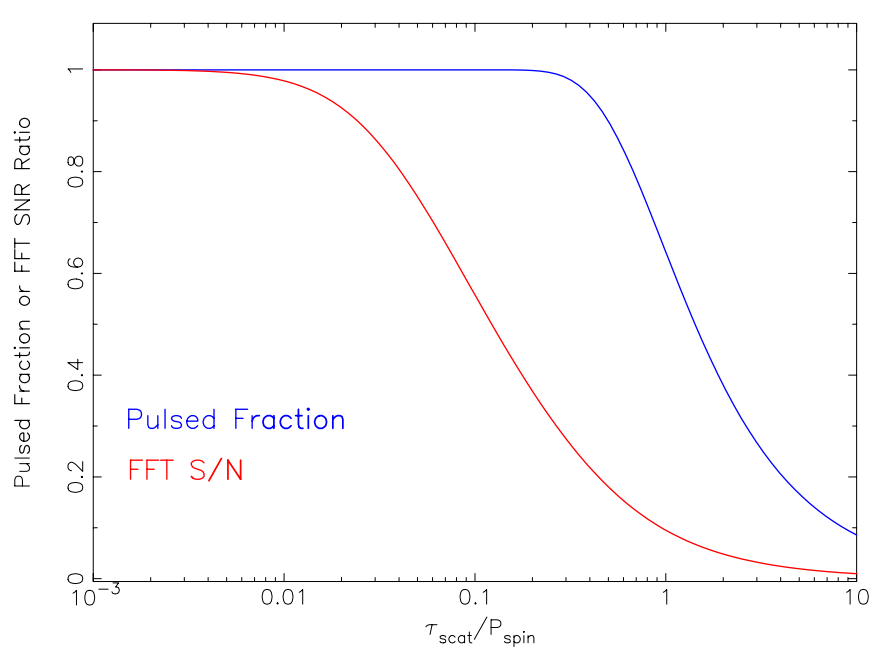

Figure 1. Effect of temporal broadening on a pulse with an intrinsic pulse width of $5 \%$ of the spin period, $P_{\text {spin }}$, assuming a one-sided exponential filter for the temporal broadening. The blue line indicates the fraction of the total pulsed flux density from the pulsar that remains pulsed after the scattering. The red line depicts the "Fourier S/N" (i.e., the sum of average noise-level normalized and mean-subtracted Fourier amplitudes) of a 16 harmonic summation of the power in a standard Fourier search. The latter indicates the detectability of pulsars in a blind survey and implicitly incorporates the pulse profile width increases and pulse shape changes caused by scattering. Most sensitivity is lost beyond $\tau_{\text {scat }} \sim 0.2 P_{\text {spin }}$

(A color version of this figure is available in the online journal.)

Next, the steepness of the pulsar emission spectrum adversely impacts searches at high frequencies. The trend from younger to older pulsars is a steepening of the spectral index, from -1 to -2 (Lorimer et al. 1995) with a mean of -1.6. Using a sample of 266 pulsars, Maron et al. (2000) find that the flux density typically has a single power law $\left(S_{v} \propto v^{\alpha}\right)$, with an average spectral index $\langle\alpha\rangle=-1.8 \pm 0.2$ (Maron et al. 2000). For this paper, we use a mean pulsar spectral index of -1.7 , a value intermediate between that derived by Lorimer et al. (1995) and Maron et al. (2000). About $10 \%$ of the sample require a dual power law, with a steeper frequency dependence above $\sim 1.5 \mathrm{GHz}$; these pulsars would be difficult to detect at high frequencies. Conversely, there exists a minority population $(<2 \%)$ of pulsars that show a flattening or even an upturn in their spectra at higher frequencies (Kramer et al. 1996; Löhmer et al. 2008).

Finally, the frequency dependences of the performance of the telescope and its receivers, the Galactic background, and the sky brightness all have an impact on the choice of the observing frequency. In the case of the GBT, the receiver contributions to the system temperature are roughly flat from 1.5 to $20 \mathrm{GHz}$. At lower frequencies $(v<10 \mathrm{GHz})$, strong emission from the GC region is a significant (and sometimes overlooked) contributor to the system temperature, with $T_{\mathrm{BG}}=340(\mathrm{v} / 2.7 \mathrm{GHz})^{-2.7} \mathrm{~K}$ (Reich et al. 1990). Conversely, the atmosphere makes a significant contribution to the system temperature at high frequencies, $\gtrsim 15 \mathrm{GHz}$, especially at the low GC elevations when observed with the GBT. The lowest GBT system temperatures can be shown to arise in the frequency range $\sim 10-18 \mathrm{GHz}$, with the upper and lower bounds determined by contributions to $T_{\text {sys }}$ from the atmosphere and the GC region, respectively.

All of the foregoing effects were combined to determine the optimal observing frequency for the GBT search for pulsars at the GC. This was done by computing the signal-to-noise ratio $(\mathrm{S} / \mathrm{N})$ for a $10 \mathrm{hr}$ GBT integration as a function of frequency (using Equation (1) of Dewey et al. 1985) for a pulsar with 


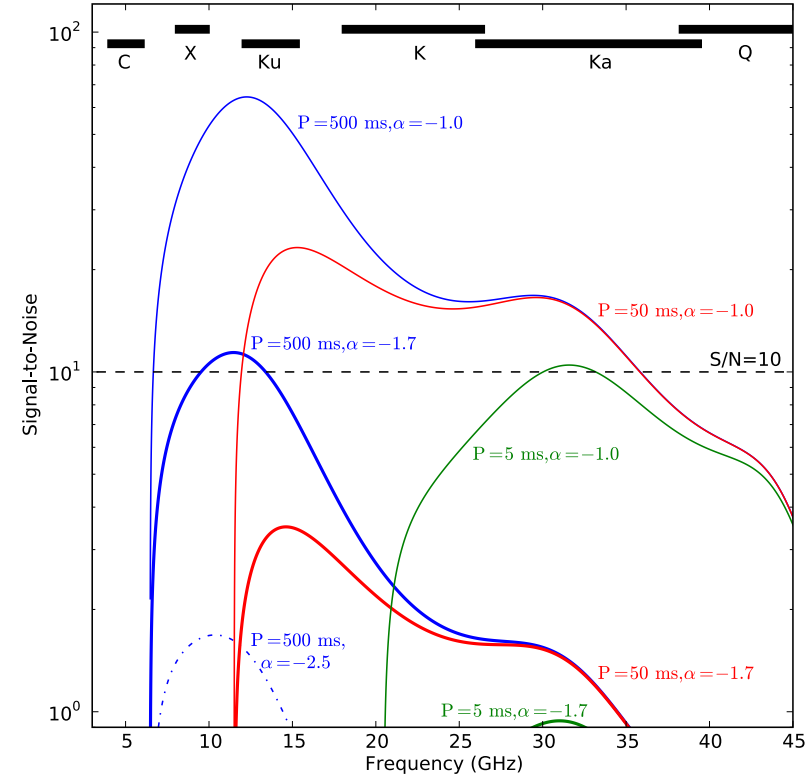

Figure 2. Frequency dependence of the detectability of pulsars at the GC for three representative periods, $P=5 \mathrm{~ms}$ (green), $50 \mathrm{~ms}$ (red), and $500 \mathrm{~ms}$ (blue) for a range of power-law spectral indices $\alpha=-1$ (thin line), -1.7 (solid line), and -2.5 (dashed line). The vertical axis gives the expected $\mathrm{S} / \mathrm{N}$ for a $10 \mathrm{hr}$ integration with the GBT at the GC, normalized to a pulsar of period $1 \mathrm{~s}$, flux density $S_{v}=1 \mathrm{mJy}$ at a frequency of $1 \mathrm{GHz}$, and whose spectrum has the frequency dependence $S_{v} \propto v^{\alpha}$. The frequency range for the current suite of GBT receivers is shown at the top of the figure.

a mean flux density of $S_{v}=1 \mathrm{mJy}$ at a frequency of $1 \mathrm{GHz}$ and an intrinsic pulsar duty cycle of $10 \%$. This was done for three representative spectral indices, $\alpha=(-1.0,-1.7,-2.5)$ (where $S_{v} \propto v^{\alpha}$ ). Finally, three representative periods $(5,50$, and $500 \mathrm{~ms}$ ) were also used to illustrate the GBT's sensitivity to different pulsar populations (corresponding, roughly, to millisecond pulsars, partially recycled or young pulsars, and normal pulsars, respectively).

The results of this analysis are summarized in Figure 2, where the $\mathrm{S} / \mathrm{N}$ for a $10 \mathrm{hr}$ GBT integration is plotted versus frequency; we use $\mathrm{S} / \mathrm{N}=10$ as the detection threshold. It is clear from the figure that, for the assumed flux density ( $1 \mathrm{mJy}$ at $1 \mathrm{GHz})$, recycled or millisecond pulsars would not be detectable with the GBT unless they have flat spectral indices $(\alpha \sim-1)$, while pulsars with $\alpha=-2.5$ would be entirely undetectable. It is also clear that the optimal search frequency for "normal" pulsars (period $\sim 500 \mathrm{~ms}$ and $\alpha \sim-1.7$ ) lies in the range $10-16 \mathrm{GHz}$, with the upper end of this frequency range also allowing the detection of shorter-period, flat-spectrum pulsars. The frequency range $12-12.8 \mathrm{GHz}$ is also affected by strong satellite-based interference at the GBT. This motivated our choice of $15 \mathrm{GHz}$ as the optimal observing frequency for the GBT search for pulsars at the GC.

\section{OBSERVATIONS, DATA ANALYSIS, AND RESULTS}

\subsection{The Observations}

The search for pulsars at the GC was carried out with the $K u$-band receiver of the GBT in the summers of 2006 and 2008, with the GBT Pulsar Spigot (Kaplan et al. 2005) used as the back end. The observations were typically restricted to GC elevations higher than $10^{\circ}$ to reduce atmospheric contributions to the system temperature $\left(T_{\text {sys }}\right)$. The GC is only visible with the GBT for $\sim 6 \mathrm{hr}$ above this elevation limit per day, and
Table 1

GBT Observational Details

\begin{tabular}{ccccc}
\hline \hline Date & $\begin{array}{c}v_{\text {obs }} \\
(\mathrm{GHz})\end{array}$ & $\begin{array}{c}\text { On-Src } \\
\text { Time }(\mathrm{hr})\end{array}$ & $\begin{array}{c}T_{\text {sys }} \\
(\mathrm{K})^{\mathrm{a}}\end{array}$ & $\begin{array}{c}\text { Sensitivity } \\
(\mu \mathrm{Jy})^{\mathrm{b}}\end{array}$ \\
\hline 2006 Jun 22 & 14.8 & 2.5 & 32 & 18 \\
2006 Jun 28 & 14.8 & 4.5 & 38 & 16 \\
2006 Jun 29 & 14.8 & 5.5 & 32 & 12 \\
2006 Aug 10 & 14.8 & 3.0 & 37 & 19 \\
2006 Aug 11 & 14.8 & 2.25 & 50 & 30 \\
2008 Aug 30 & 14.4 & 4.25 & 36 & 16 \\
2008 Aug 31 & 14.4 & 5.5 & 33 & 13 \\
\hline
\end{tabular}

Notes.

a The quoted $T_{\text {sys }}$ values are the averages for each run, after correcting for the different elevations of the GC and the calibrators.

$\mathrm{b}$ The last column lists the $10 \sigma$ detection threshold flux density for pulsars with a duty cycle of $10 \%$ in each individual observing session. Note that the final searches combined data from adjacent days, and hence had a lower detection threshold flux density, $\sim 10 \mu \mathrm{Jy}$, apiece for the runs of 2006 June 28 and 29 and 2008 August 30 and 31 .

the observations were therefore broken up into multiple short sessions. While attempts were made to group these sessions as close together as possible (so as to combine multiple sessions in a search), this was often not possible due to poor high-frequency observing weather.

The 2006 observing runs were on June 22, 28, and 29, July 10, and August 10 and 11 . The July 10 data were affected by strong broadband radio frequency interference (RFI) due to an overloaded network switch, and will not be discussed further; data from the other runs were not affected by this issue. The 2008 observations were on August 30 and 31, with each run preceded by a $5 \mathrm{hr}$ observation of a blank field (J2000 co-ordinates: R.A. $=11^{\mathrm{h}} 46^{\mathrm{m}} 08^{\mathrm{s}} .1$, decl. $=-27^{\mathrm{d}} 47^{\prime} 32^{\prime \prime} .9$ ), with the same observing parameters as for the GC; the latter served to test for systematic effects in the data. The on-source times for the GC are listed in Column 3 of Table 1.

All observing runs included short observations of strong known pulsars (B1800-21 in most of the 2006 runs and the magnetar, XTE J1810-197, in 2008 and 2006 August), to test that the system was working properly; these were clearly detected in every run.

All observing sessions used the maximum allowed Spigot bandwidth of $800 \mathrm{MHz}$, sub-divided into 1024 channels, with two circular polarizations, three-level sampling, and a dump rate of $81.92 \mu \mathrm{s}$. The $800 \mathrm{MHz}$ band was centered at $14.8 \mathrm{GHz}$ in the 2006 runs and at $14.4 \mathrm{GHz}$ in the 2008 runs; the shift in the central frequency was to exclude some RFI (at $14.88 \mathrm{GHz}$ ) from the observing band. In 2006, an automatic level controller was used to hold the power levels fixed on the GC. Unfortunately, this was found to itself result in intermittent power jumps, and we hence chose to not use this feature in 2008. In all runs, observations of strong nearby calibrators (or the GC itself) were used to correct the telescope pointing and surface, typically every $2-3 \mathrm{hr}$. System temperatures were measured on the calibrators by firing a noise diode, and ranged between $26 \mathrm{~K}$ and $40 \mathrm{~K}$ on most observing runs; however, the short session on August 11 had $T_{\text {sys }} \sim 50 \mathrm{~K}$ on the calibrator, due to overcast conditions. After taking into account the slightly different elevations of the $\mathrm{GC}$ and the calibrators, the estimated average system temperatures toward the GC were $32-38 \mathrm{~K}$ (again, except on August 11 where $T_{\text {sys }} \sim 50 \mathrm{~K}$ ). The gain of the $K u$-band receiver is $1.5 \mathrm{~K} \mathrm{Jy}^{-1}$. Finally, the size of the GBT beam at $14.8 \mathrm{GHz}$ is $\sim 50^{\prime \prime}$, corresponding to a spatial 
radius of $\sim 1 \mathrm{pc}$ at the distance of the GC $\left(7.9_{-0.7}^{+0.8} \mathrm{kpc}\right.$; Reid et al. 2009).

Table 1 presents a summary of the observational details: the columns are (1) the observing date, (2) the central frequency, (3) the GC on-source time, (4) the typical system temperature on the GC during the run, and (5) the $10 \sigma$ detection threshold for pulsars with a duty cycle of $10 \%$ in each session (using Equation (1) of Dewey et al. 1985). As discussed below, the final searches were carried out on the combined data sets from (1) 2006 June 28 and 29 ( 10 hr), (2) 2006 August 10 and 11 ( $\sim 5.25 \mathrm{hr}$ ), and (3) 2008 August 30 and 31 ( $\sim 9.75 \mathrm{hr})$. The first and third of these had the best sensitivity, with $10 \sigma$ detection thresholds of $\sim 10 \mu \mathrm{Jy}$ each, again assuming a pulsar duty cycle of $10 \%$.

\subsection{Data Analysis}

The observations were reduced and analyzed with the PRESTO $^{8}$ software package (Ransom 2001). Initially, all data were time-domain clipped at the $6 \sigma$ level to remove any strong RFI. As noted above, the 2006 data suffered from random jumps in the power level due to an automatic level controller in the signal path. The effect of these jumps was removed by forcing data between jumps to a common mean of zero, with data in the immediate vicinity of the jump points edited out. Data from successive days (2006 June 28 and 29, 2006 August 10 and 11, and 2008 August 30 and 31) were combined into a single data stream in order to enhance the detectability of faint pulsars during the periodicity search. The data from 2006 June 28 and 29 and 2006 August 10 and 11 were also analyzed independently to examine the possibility of intermittent signals. The data streams were de-dispersed for dispersion measures of 1500, 2000, 2500, $3000,3500,4000$, and $5000 \mathrm{~cm}^{-3}$ pc. Finer sampling in dispersion measure is not required for the detection of slow pulsars because the relative dispersion delay across the $800 \mathrm{MHz}$ band at $14.8 \mathrm{GHz}$ is only $2 \mathrm{~ms}$ for every $1000 \mathrm{~cm}^{-3} \mathrm{pc}$. Note that our search was only sensitive to pulsars with periods $<2 \mathrm{~s}$, due to power fluctuations associated with atmospheric variability that reduced our sensitivity and precluded a search for pulsed emission at longer periods.

The search for pulsars was carried out using standard techniques: the signal was Fourier transformed, a red-noise reduction was applied to the spectrum, and the PRESTO algorithm accelsearch (Ransom et al. 2002) used to identify potential pulsars. The original time series were then folded according to the pulse characteristics of each candidate in order to determine the significance of each signal, construct its pulse profile, and refine its characteristics (e.g., period, period derivative, etc.)

We also separately searched the 2008 data for bright individual pulses, like those seen from young pulsars such as the Crab, using the program single_pulse.py from PRESTO, a timedomain matched-filtering technique similar to that described by Cordes \& McLaughlin (2003). The de-dispersed time series were first down-sampled by a factor of 10 to an effective time resolution of $0.8192 \mathrm{~ms}$. We then convolved the data with square-wave pulses of a variety of widths from 1 to 150 samples in duration and searched for peaks substantially above those of the noise variations. In no case did we find strong pulses that were obviously dispersed in nature and could be clearly distinguished from RFI. Of course, a significantly larger observing bandwidth would provide not only more sensitivity to pulses of this sort, but would also dramatically increase the amount of dis-

\footnotetext{
8 See http://www.cv.nrao.edu/ sransom/presto/.
}

2 Pulses of Best Profile
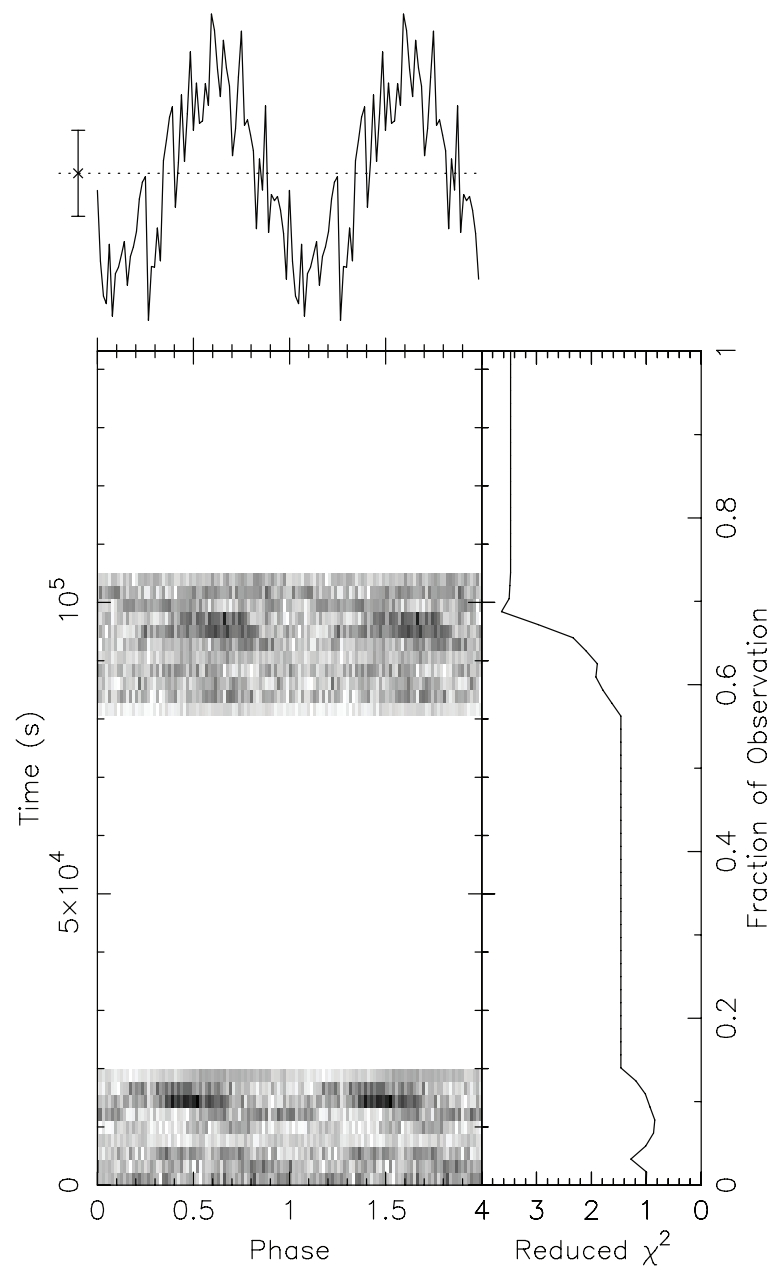

Figure 3. Detection plot of the putative $607 \mathrm{~ms}$ pulsar candidate from the data set of 2006 June 28-29; the top panel shows the final pulse profile, while the bottom panel shows the growth of reduced $\chi^{2}$ with increasing data. The gray scale denotes the signal strength, with darker signals indicating a stronger signal. The white regions correspond to time intervals during which the telescope was not pointing at the GC.

persive smearing across the band, allowing us to use dispersion to rule out pulses of terrestrial origin. While we also searched the 2006 data for single-pulse signals without any detections, the sharp jumps introduced by the auto-leveler in these data sets rendered it particularly difficult to distinguish astronomical pulses from system-generated signals.

\subsection{Results}

Our original search in 2006 yielded candidate pulsed signals of high single-trial statistical significance $(>8 \sigma)$ in the June 28-29 and August 10-11 data sets independently. The highest $\mathrm{S} / \mathrm{N}$ was obtained at a trial dispersion measure of $3000 \mathrm{pc} \mathrm{cm}^{-3}$ and a period of $607 \mathrm{~ms}$ in the data sets of 2006 June 28-29 (11 $\sigma$ significance); this pulse profile is shown in Figure 3. Signals at the harmonic periods of $303 \mathrm{~ms}$ and $152 \mathrm{~ms}$ were also weakly detected in these data sets, typically at $\sim 4 \sigma$ significance. A similar signal, with a period of $\sim 604 \mathrm{~ms}$, was independently detected in the data sets of August $10-11$, with a significance of $\sim 8 \sigma$ at trial dispersion measures of 3000-4000 pc cm${ }^{-3}$; this is shown in Figure 4. Again, harmonics of this signal (at $302 \mathrm{~ms}$ and $151 \mathrm{~ms}$ ) were detected at 
2 Pulses of Best Profile
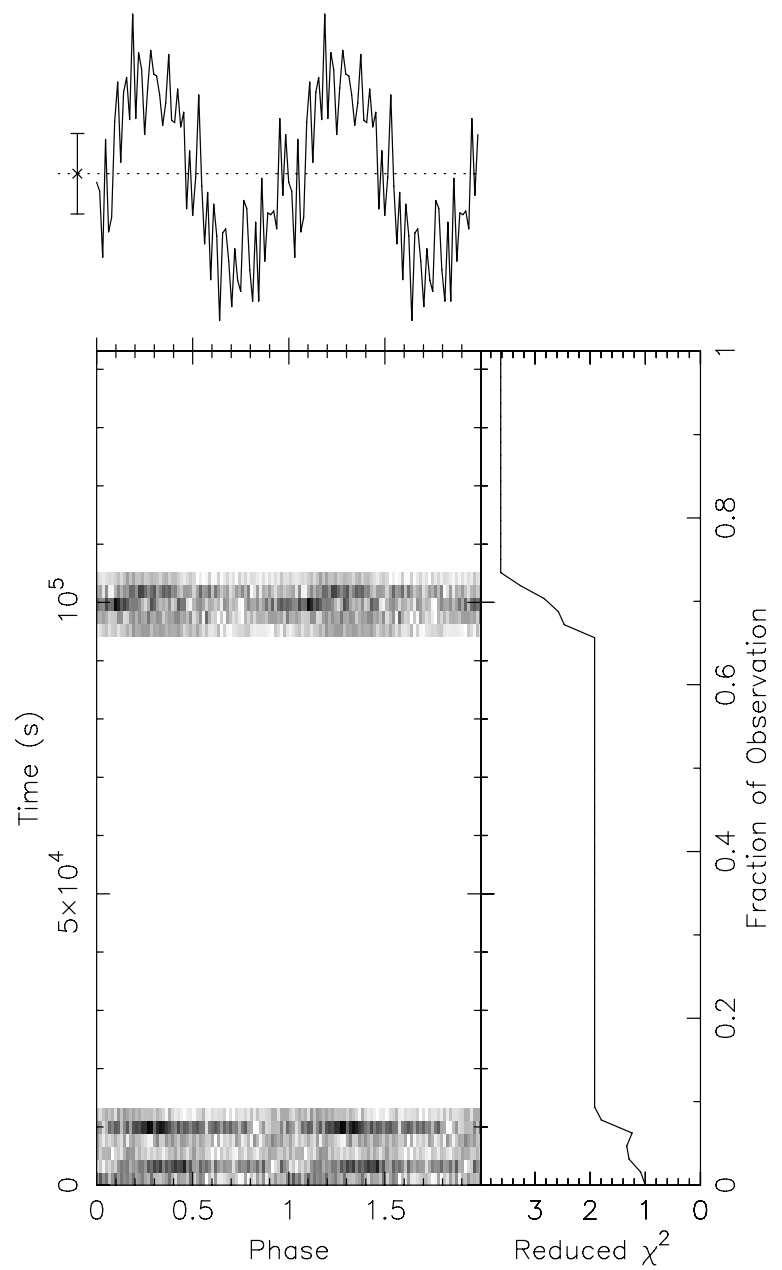

Figure 4. Detection plot of the putative $604 \mathrm{~ms}$ candidate from the data sets of 2006 August 10-11.

lower significance $(\sim 3.5 \sigma)$. The $607 \mathrm{~ms}$ signal was also seen on folding the data from 2006 June 22 using the solutions determined from the data sets of June 28-29; this yielded a detection significance of $18 \sigma$ and the pulse profile of Figure 5. We also tested that these candidate pulsed signals do not arise from periodicities in the power jumps in the 2006 data.

No evidence for a pulsed signal was found in the data sets from 2008 at these, or any other, periods. The 2008 data were also folded at the pulse periods of the candidates detected in 2006 ( 604-607 ms), without any detectable signal.

Finally, no other pulsar candidates (above $6 \sigma$ significance) were found in any of the data sets. The noise levels achieved in each observation are listed in Table 1 . The best sensitivity was achieved with the data sets of 2006 June 28-29, and 2008 August $30-31$, which yielded a $10 \sigma$ detection threshold of $10 \mu \mathrm{Jy}$, assuming a pulsar duty cycle of $10 \%$.

\section{DISCUSSION AND CONCLUSIONS}

\subsection{The 607 ms Candidate of 2006}

The high statistical significance of the pulsed signal detected in the data sets of 2006 implies that it arises either from a genuine pulsar toward the GC or as an artifact of a terrestrial signal (e.g., RFI). While the non-detection in the 2008 data sets
2 Pulses of Best Profile
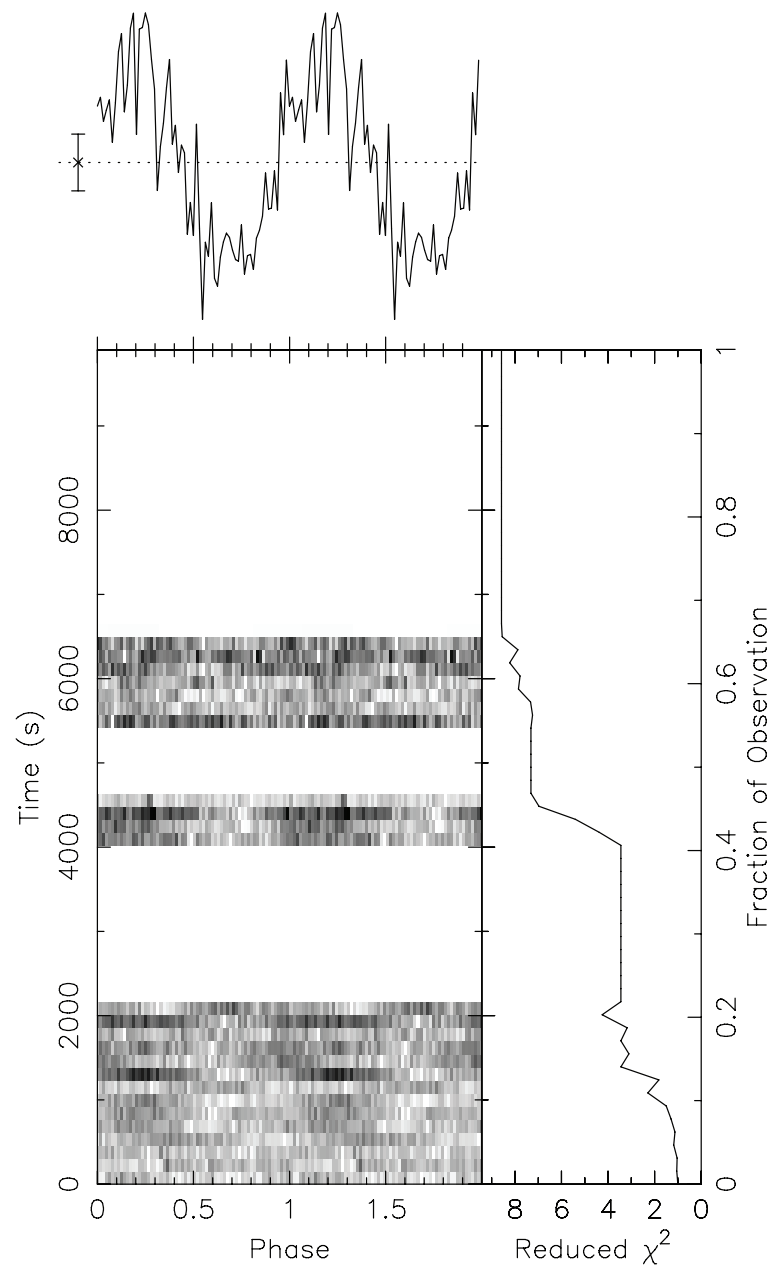

Figure 5. Detection plot of the pulsed signal on 2006 June 22, after folding at the pulse period of the candidate detected in the data sets of 2006 June 28-29.

might indicate the latter possibility, it should be emphasized that the GC environment is very different from the environments of typical pulsars. Specifically, a pulsar on a short-period $(<100$ year) orbit around the GC could easily have its emission beam precess away from our line of sight over a timescale of two years. This implies that caution must be used while dismissing possible pulsar candidates toward the GC, although one should retain skepticism about their reality. We will hence summarize the characteristics of the pulsed signals seen in the 2006 data sets, and discuss the possibilities that they might arise from a real pulsar or RFI.

Figures 3 and 4 show that the detection $\chi^{2}$ in the two long data sets of 2006 increases steadily over the course of each observing session, indicating that, if the signals are spurious or local RFI, they are at least persistent both over the course of each observation and over multiple observing epochs. It is curious, however, that the period of the pulsed signal is different in the sessions on June 28-29 and August 10-11; for RFI, this would require either that we have detected two distinct but alternately intermittent RFI signals, or that the period of the RFI itself is changing. Interpreted in terms of Doppler shifts, the period change corresponds to a velocity change of $1500 \mathrm{~km} \mathrm{~s}^{-1}$, much larger than that associated with Earth's motion around the Sun or the motions of terrestrial objects, but not implausible for a pulsar orbiting around the GC. 
Next, the $\mathrm{S} / \mathrm{Ns}$ of our candidates peak at dispersion measures of $\approx 3000-4000 \mathrm{pc} \mathrm{cm}^{-3}$, comparable to values expected for pulsars at the GC. Unfortunately, our small fractional bandwidth means that the dispersion in the signal across the band is very small ( $\sim 3.6 \mathrm{~ms}$ over the $800 \mathrm{MHz}$ bandwidth). This means that, unlike the situation in low-frequency pulsar surveys, dispersion cannot be used to test whether the signal is of extraterrestrial origin. Finally, the pulse profiles of the candidates are extremely broad, with a duty cycle of $\sim 50 \%$, unlike the narrow profiles expected for high-frequency pulsar emission. However, the pulse properties, too, could be affected by the unique GC environment. For example, the thin-screen approximation might not be applicable for the scattering, or the screen could be much closer to the pulsar than typical estimates of $\sim 100 \mathrm{pc}$; both of these would increase the scattering time and broaden the pulse profile, even at such a high frequency. Specifically, the scattering timescale at a frequency of $14.6 \mathrm{GHz}$ for an object at the GC is $2.5 / D_{\text {scat }} \mathrm{s}$, where $D_{\text {scat }}$ is the distance, in pc, of the scattering medium from Sgr A*. While the best estimate of $D_{\text {scat }}$ is $\sim 100$ pc from angular broadening measurements of Sgr A* and nearby masers (Cordes \& Lazio 1997), the effect of scattering material close to $\mathrm{Sgr} \mathrm{A}^{*}$ is much stronger on temporal smearing than on the angular broadening of background sources. As such, the angular broadening estimates of $D_{\text {scat }}$ do not rule out a substantial contribution to the pulse broadening from material closer to $\mathrm{Sgr} \mathrm{A}^{*}$. One may hence have a sizeable contribution to the pulse broadening from material at $D_{\text {scat }} \lesssim 10$ pc (e.g., Macquart \& Bower 2006). The expected temporal smearing timescale would then be $\gtrsim 250 \mathrm{~ms}$, comparable to that needed to explain the pulse shape of the $607 \mathrm{~ms}$ candidate. The large observed duty cycle of the candidate thus does not rule out the possibility that the signal arises from a genuine pulsar.

It thus appears very difficult to rule out the reality of the candidate on the basis of the 2006 data alone, and, as noted above, the non-detection in 2008 could arise due to precession of the pulsar beam away from our sightline. Thus, while we remain skeptical about the reality of these signals, we conclude that further observations are needed to test the possibility that they arise from a genuine pulsar at the GC.

\subsection{Constraints on the GC Pulsar Population}

There is compelling but indirect evidence for a substantial population of neutron stars at the GC. However, strong interstellar scattering along the line of sight has limited past searches for radio pulsars. To overcome these effects, we have used the superb sensitivity of the GBT to carry out a deep search for pulsars in the central parsec of the $\mathrm{GC}$ at $15 \mathrm{GHz}$ - the highest observing frequency at which a search has been carried out to date. Despite this, we find no convincing pulsar candidates. Was our survey sufficiently sensitive to detect a population of pulsars around Sgr $\mathrm{A}^{*}$ ?

The total number of pulsars detectable at the GC depends on the total number of pulsars accumulated in the region, and the fraction of these objects that would be detectable given our survey sensitivity, and the S/N considerations of Section 2. The detectable fraction depends particularly on the number of pulsars with flat spectral indices, since these objects influence the pulsar luminosity function most strongly at frequencies $>10 \mathrm{GHz}$ where they are most easily detectable toward Sgr A*.

A simple estimate of the number of detectable pulsars can be obtained by positing that the Sgr A* pulsar population has similar properties to those of the known population of pulsars and to estimate the fraction of the known population

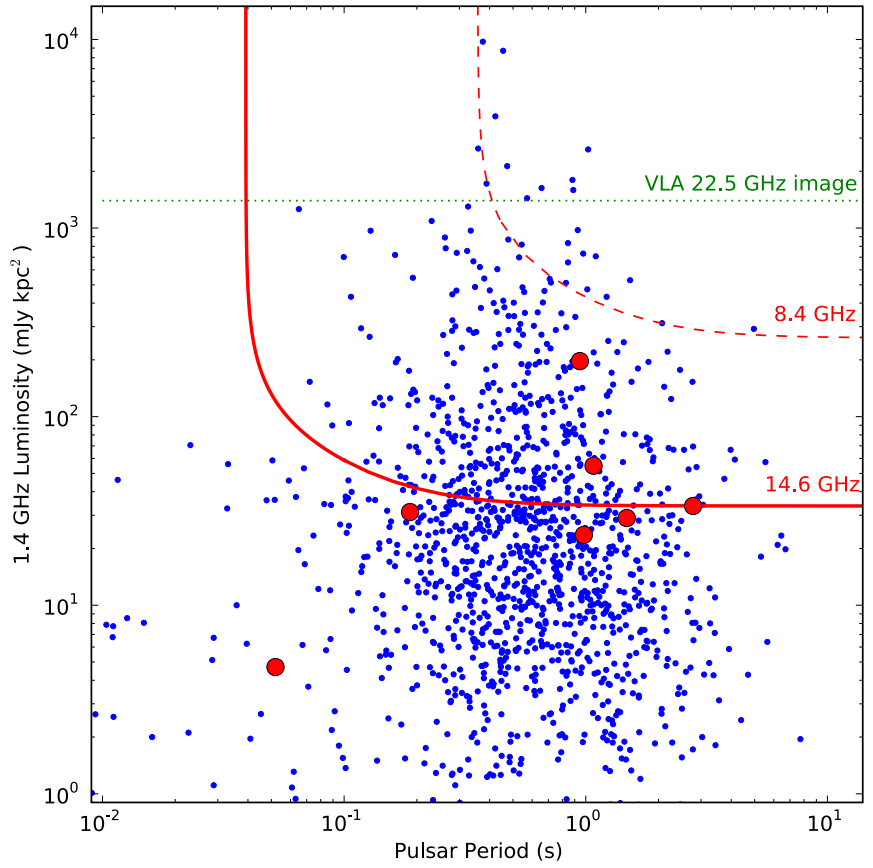

Figure 6. 1.4 GHz luminosities of the known sample of pulsars vs. pulse periods (blue dots). Larger circles (red dots) indicate those pulsars within $1^{\circ}$ radius of Sgr A*. The $10 \sigma$ pulsar sensitivity of our $14.6 \mathrm{GHz}$ search is shown by the solid red line. This was obtained by using the flux density limit $(10 \mu \mathrm{Jy}$ at $10 \sigma)$ of our survey to calculate the luminosity limit at the distance of the GC and then scaling the result to a frequency of $1.4 \mathrm{GHz}$, using an average spectral index of $\langle\alpha\rangle=-1.7$. We also show sensitivity curves derived in the same manner for a Parkes $8.4 \mathrm{GHz}$ pulsar survey of the GC (the dashed red line, with a $10 \sigma$ detection threshold of $200 \mu \mathrm{Jy}$; Johnston et al. 2006), and a deep $22.5 \mathrm{GHz}$ VLA image of the GC (the dotted green line, with a $10 \sigma$ detection threshold of $200 \mu$ Jy; Zhao et al. 2009).

that would be detectable at the GC with our survey. This is shown in Figure 6, where we have plotted pulsars with measured 1.4 GHz luminosities (from the Manchester et al. 2005 catalog) on a period-luminosity diagram. The solid red line shows the pulsar sensitivity curve of our $14.6 \mathrm{GHz}$ survey, obtained using Equations (1)-(3) with a $10 \sigma$ detection threshold of $10 \mu \mathrm{Jy}$, and assuming a $10 \%$ pulsar duty cycle, a scattering screen distance $D_{\text {scat }}=133 \mathrm{pc}$ (Section 2), and a GC dispersion measure of $1700 \mathrm{pc} \mathrm{cm}^{-3}$. The sensitivity curve has been scaled to $1.4 \mathrm{GHz}$ using a mean pulsar spectral index of -1.7 . The cutoff in period where most of the sensitivity is lost is taken to be at $P_{\text {spin }}=2 \times \tau_{\text {scat }}$. This is less severe than the scatterbased sensitivity cutoff in Figure 1 but does reflect the fact that some partially recycled or young pulsars (i.e., $P<50 \mathrm{~ms}$ ) would be detectable if they were much brighter than our noise threshold.

For comparison purposes, this figure also shows the $8.4 \mathrm{GHz}$ sensitivity curve for the Parkes GC survey (Johnston et al. 2006), and the $5 \sigma$ noise threshold for a deep imaging survey of the GC at $22.5 \mathrm{GHz}$, using the Very Large Array (VLA; Zhao et al. 2009), again scaling both of these to a frequency of $1.4 \mathrm{GHz}$ using a mean spectral index of -1.7 . We also highlight the seven known pulsars within $1^{\circ}$ of $\mathrm{Sgr} \mathrm{A}^{*}$, including four new ones from Deneva et al. (2009) and Camilo et al. (2009).

A more rigorous estimate can be obtained by computing the fraction of pulsars detectable above some flux density cutoff by considering the pulsar luminosity function at $v_{0}=1.4 \mathrm{GHz}$, $f_{0}(L)$, combined with the spectral index distribution, $p(\alpha)$. This is the approach followed by Pfahl \& Loeb (2004) and Cordes \& 
Lazio (1997), but updated with the most recent results on pulsar luminosity functions (Faucher-Giguère \& Kaspi 2006; Lorimer et al. 2006). We model the $1.4 \mathrm{GHz}$ luminosity function as a power law between lower and upper cutoffs $L_{\min }$ and $L_{\max }$, respectively:

$$
f_{0}(L)=A L^{-\beta}, \quad A=(1-\beta)\left[L_{\max }^{1-\beta}-L_{\min }^{1-\beta}\right]^{-1},
$$

where the normalization is chosen so that the integral over all luminosities is unity, such that $f d L$ is interpreted as the fraction of all pulsars with luminosities between $L$ and $L+d L$. Recent studies suggest that $L_{\text {min }}=0.01 \mathrm{mJy} \mathrm{kpc}^{2}, L_{\max }=32 \mathrm{Jy} \mathrm{kpc}^{2}$, and $\beta=1.2-2$ (Faucher-Giguère \& Kaspi 2006; Lorimer et al. 2006). Following Smits et al. (2009), the spectral index distribution is modeled as a Gaussian

$$
p(\alpha)=\frac{1}{\sqrt{2 \pi \sigma_{\alpha}^{2}}} \exp \left[-\frac{\left(\alpha-\alpha_{m}\right)^{2}}{2 \sigma_{\alpha}^{2}}\right],
$$

with mean spectral index $\alpha=-1.7$ and standard deviation $\sigma_{\alpha}=0.35$. then

The pulsar luminosity function at some arbitrary frequency is

$$
f(v, L)=\int_{-\infty}^{\infty} d \alpha p(\alpha) f\left(L\left(\frac{v}{v_{0}}\right)^{-\alpha}\right),
$$

where, for a given spectral index $\alpha$ chosen from the distribution, the luminosity function $f$ has lower and upper cutoffs

$$
L_{\min }^{\prime}=L_{\min }\left(\frac{v}{v_{0}}\right)^{\alpha}, \quad L_{\max }^{\prime}=L_{\max }\left(\frac{v}{v_{0}}\right)^{\alpha},
$$

and the normalization constant $A$ is modified to

$$
A=(1-\beta)\left(\frac{v}{v_{0}}\right)^{-\alpha \beta}\left(L_{\max }^{\prime 1-\beta}-L_{\min }^{\prime 1-\beta}\right)^{-1}
$$

in order to ensure that $f d L$ may be interpreted as the fraction of all pulsars in the luminosity range $L$ to $L+d L$. We integrate Equation (5) to obtain the total fraction of pulsars above a given flux density threshold $L_{\text {cut }}=d^{2} S_{\text {cut }}$. Of course, this threshold depends on both the pulsar period and observing frequency due to propagation effects and changes in the system temperature. Figure 7 hence plots the fraction of the pulsar population detectable above $10 \sigma$ significance at $15 \mathrm{GHz}$ versus pulse period.

Note that the present survey is sensitive only to the slower $(P>40 \mathrm{~ms})$ and more-luminous $\left(L>40 \mathrm{mJy} \mathrm{kpc}{ }^{2}\right)$ pulsars. The millisecond pulsars that are presumably powering the lowmass X-ray binaries near Sgr A* (Muno et al. 2005) and the low-luminosity tail of young pulsars (Camilo et al. 2009) are out of the reach of our survey. However, it is also clear from the figures that this is the first survey capable of peering past the "fog" of scattering material and detecting a significant number of pulsars within a parsec of the GC with properties similar to the known pulsar population. Past high-frequency pulsar searches (Johnston et al. 2006) or imaging searches (Zhao et al. 2009) have not had the requisite temporal or flux density sensitivity to detect a significant fraction of the known population. The GBT search thus represents a significant improvement over past pulse searches and imaging efforts. We note, in passing, that this implicitly assumes that pulsar spectral indices do not typically steepen at high frequencies, $\gtrsim 5 \mathrm{GHz}$.

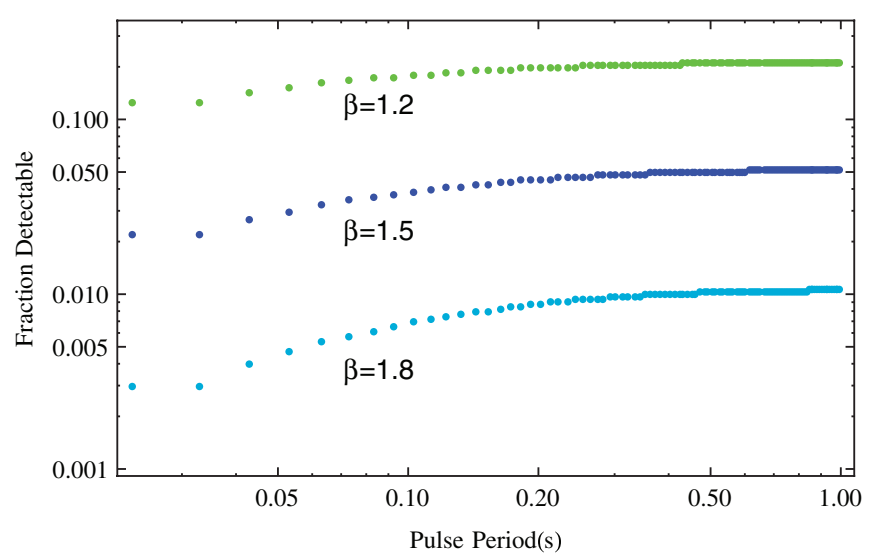

Figure 7. Fraction of GC pulsars that would be detectable at $10 \sigma$ significance for a $10 \mathrm{hr}$ integration on the $\mathrm{GBT}$ at $15 \mathrm{GHz}$ as a function of spin period. We assume an intrinsic pulse width of $10 \%$ of the spin period and take $D_{\text {scat }}=100 \mathrm{pc}$. The curves, from top to bottom, correspond to luminosity function indices of $\beta=1.2,1.5$, and 1.8, with a mean spectral index of $\alpha=-1.6$ and dispersion $\sigma_{\alpha}=0.35$, as discussed in the text.

(A color version of this figure is available in the online journal.)

It is clear from Figures 6-7 that the $15 \mathrm{GHz}$ GBT survey could have detected a significant fraction $(\sim 1 \%-15 \%)$ of the pulsars around $\operatorname{Sgr} \mathrm{A}^{*}$, if they had properties similar to those of the known population. The estimate obtained from Figure 6 is at the high end (15\%) and is possibly biased by luminositydependent completeness limits in pulsar surveys. The lowest estimate $(\sim 1 \%)$ comes from the curve in Figure 7 with the steepest luminosity slope $(\beta=1.8)$. We adopt a nominal value of $5 \%$ from the intermediate curve $(\beta=1.5)$ which is based on our best current knowledge of the properties of the pulsar population and the scattering material toward Sgr A*. Given this detection fraction and our null detection, we can use straightforward binomial statistics to estimate the size of the putative pulsar population at the GC. If the probability of detecting a normal pulsar is $5 \%$, the non-detection of any pulsars in our survey implies the upper limit (at $99 \%$ confidence level) of 90 normal pulsars within the $1 \mathrm{pc}$ region around the GC encompassed by the GBT beam. Taking the full allowed range of the detection fraction $(1 \%-15 \%)$, the upper limit on the number of normal pulsars ranges from 460 to 30, respectively, again at $99 \%$ confidence level. Although our estimate is both approximate and subject to much uncertainty, we note that it is significantly lower than the $\sim 100-1000$ pulsars derived by Pfahl \& Loeb (2004) for the normal pulsar population with orbits of $\leqslant 100 \mathrm{yr}$ (i.e., a radius 50 times smaller than the size of the GBT search area).

Finally, we have shown that the frequency range $10-16 \mathrm{GHz}$ is optimal for searches for "normal" pulsars at the GC. The GBT remains the most powerful high-frequency instrument capable of detecting the GC pulsar population for at least the next decade, until the advent of next-generation telescopes like the Square Kilometer Array. The primary limitation of the GBT (and of the present survey) is the relatively small instantaneous bandwidth $(800 \mathrm{MHz})$ available for such searches, resulting in a small fractional bandwidth. An increased bandwidth at the GBT would imply not only an improvement in sensitivity, but also a better rejection of terrestrial signals, using the dispersive sweep of genuine signals across the band. Figure 6 shows that an improvement in sensitivity by merely a factor of 2-3 would push the GBT into the bulk of the pulsar population. Future GBT experiments should hence aim to utilize the full frequency 
coverage available with the high-frequency receivers, for both better discrimination against systematic effects and improved sensitivity.

The Green Bank Telescope is operated by the National Radio Astronomy Observatory, a facility of the National Science Foundation operated under cooperative agreement by Associated Universities, Inc. We thank Carl Bignell for his extraordinary efforts to schedule this project on the GBT. N.K. is grateful for support from the Max-Planck Society and the Alexander von Humboldt Foundation, as well as from a Ramanujan Fellowship. N.K. and J.P.M. also acknowledge support from NRAO Jansky Fellowships.

Facilities: GBT

\section{REFERENCES}

Bower, G. C., Roberts, D. A., Yusef-Zadeh, F., Backer, D. C., Cotton, W. D., Goss, W. M., Lang, C. C., \& Lithwick, Y. 2005, ApJ, 633, 218

Camilo, F., Ng, C., Gaensler, B. M., Ransom, S. M., Chatterjee, S., Reynolds, J., \& Sarkissian, J. 2009, ApJ, 703, L55

Cordes, J. M., Kramer, M., Lazio, T. J. W., Stappers, B. W., Backer, D. C., \& Johnston, S. 2004, New Astron. Rev., 48, 1413

Cordes, J. M., \& Lazio, J. T. W. 1997, ApJ, 475, 557

Cordes, J. M., \& McLaughlin, M. A. 2003, ApJ, 596, 1142

Deneva, J. S., Cordes, J. M., \& Lazio, T. J. W. 2009, ApJ, 702, L177

Dewey, R. J., Taylor, J. H., Weisberg, J. M., \& Stokes, G. H. 1985, ApJ, 294, L25

Faucher-Giguère, C.-A., \& Kaspi, V. M. 2006, ApJ, 643, 332

Ghez, A. M., Salim, S., Hornstein, S. D., Tanner, A., Lu, J. R., Morris, M., Becklin, E. E., \& Duchêne, G. 2005, ApJ, 620, 744
Johnston, S., Kramer, M., Lorimer, D. R., Lyne, A. G., McLaughlin, M., Klein, B., \& Manchester, R. N. 2006, MNRAS, 373, L6

Johnston, S., Walker, M. A., van Kerkwijk, M. H., Lyne, A. G., \& D’Amico, N. 1995, MNRAS, 274, L43

Kaplan, D. L., et al. 2005, PASP, 117, 643

Kramer, M., Backer, D. C., Lazio, T. J. W., Stappers, B. W., \& Johnston, S. 2004, New Astrn. Rev., 48, 993

Kramer, M., Xilouris, K. M., Jessner, A., Wielebinski, R., \& Timofeev, M. 1996, A\&A, 306, 867

Lazio, T. J. W., \& Cordes, J. M. 1998, ApJ, 505, 715

Löhmer, O., Jessner, A., Kramer, M., Wielebinski, R., \& Maron, O. 2008, A\&A, 480, 623

Lorimer, D. R., Yates, J. A., Lyne, A. G., \& Gould, D. M. 1995, MNRAS, 273, 411

Lorimer, D. R., et al. 2006, MNRAS, 372, 777

Macquart, J., \& Bower, G. C. 2006, ApJ, 641, 302

Manchester, R. N., Hobbs, G. B., Teoh, A., \& Hobbs, M. 2005, AJ, 129, 1993

Maron, O., Kijak, J., Kramer, M., \& Wielebinski, R. 2000, A\&AS, 147, 195

Muno, M. P., Pfahl, E., Baganoff, F. K., Brandt, W. N., Ghez, A., Lu, J., \& Morris, M. R. 2005, ApJ, 622, L113

Paumard, T., et al. 2006, ApJ, 643, 1011

Pfahl, E., \& Loeb, A. 2004, ApJ, 615, 253

Ransom, S. M. 2001, PhD thesis, Harvard Univ.

Ransom, S. M., Eikenberry, S. S., \& Middleditch, J. 2002, AJ, 124, 1788

Reich, W., Fuerst, E., Reich, P., \& Reif, K. 1990, A\&AS, 85, 633

Reid, M. J., Menten, K. M., Zheng, X. W., Brunthaler, A., \& Xu, Y. 2009, ApJ, 705,1548

Schödel, R., Ott, T., Genzel, R., Eckart, A., Mouawad, N., \& Alexander, T. 2003, ApJ, 596, 1015

Smits, R., Lorimer, D. R., Kramer, M., Manchester, R., Stappers, B., Jin, C. J., Nan, R. D., \& Li, D. 2009, A\&A, 505, 919

Wang, Q. D., Lu, F. J., \& Gotthelf, E. V. 2006, MNRAS, 367, 937

Wex, N., \& Kopeikin, S. 1999, ApJ, 513, 388

Zhao, J.-H., Morris, M. R., Goss, W. M., \& An, T. 2009, ApJ, 699, 186 